\section{A NOVEL PRODUCER OF THE ANTIBIOTIC KIRROMYCIN BELONGING TO THE GENUS Actinoplanes}

Sir:

In the course of a screening program for actinomycetes which produce antibacterial antibiotics, we repeatedly isolated aurodox-type antibiotics which were active against Neisseria caviae ATCC 14659 and inactive against Staphylococcus aureus ATCC 6538. These antibiotics, which act upon elongation factor $\mathrm{Tu}$, have a characteristic spectrum of antibacterial activity: they inhibit anaerobes, neisseriae and streptococci ${ }^{1 \sim 4)}$, but are ineffective against $S$. aureus ${ }^{5}$.

The producing strains we isolated were mainly Streptomyces spp. occurring with a frequency of about one producer found for every three thousand strains screened. Among the sixteen antibiotics belonging to the aurodox-type class (Table 1), aurodox $^{5)}$, kirromycin $^{6)}, \mathrm{A} 73 \mathrm{~A}^{7)}$, factumycin ${ }^{8)}$ and kirrothricin ${ }^{9)}$ occurred more frequently. We also observed that the streptomycetes co-produced humidin-type antibiotics. These macrolides, which often contaminate preparations of the aurodox-type antibiotics, exhibit high toxicity on both prokaryotic and eukaryotic cells ${ }^{10 \sim 14}$. Humidin-type antibiotics were found mainly adsorbed onto the mycelium of the producing strains and were determined in crude extracts from their UV spectrum and their toxic effect on yeast cells.

From a soil sample collected in India, we isolated an Actinoplanes strain, A8924, which produced kirromycin and did not produce any humidin-type antibiotic. Identification of the antibiotic was made using ${ }^{1} \mathrm{H}$ NMR and LC-MS ${ }^{15}$.

Strain A8924 typically had a bright orange ${ }^{16}$ ) vegetative mycelium composed of twisted hyphae with a diameter of $1 \sim 1.5 \mu \mathrm{m}$, bearing on the surface multispored spherical sporangia with a diameter of $9 \sim 13 \mu \mathrm{m}$ (Fig. 1). The sporophores are straight to slightly curved about $8 \sim 15 \mu \mathrm{m}$ long and $2 \mu \mathrm{m}$ in diameter. The oval spores, $1.5 \sim 2 \mu \mathrm{m}$ in diameter, are highly motile by means of several flagella arranged in polar tufts.

Chemotaxonomic assessment of strain A8924 revealed meso-diaminopimelic acid and glycine as distinguishing components of the cell wall ${ }^{17)}$. Xylose and arabinose were the major sugars in the whole cell hydrolysate ${ }^{18)}$. According to the classification of LeCHEVALIER and LeCHEVALIER ${ }^{19)}$, this is a cell wall'type IID.

On the basis of both its morphological and chemotaxonomic characteristics, strain A8924 was assigned to the genus Actinoplanes. There are no other examples reported in the literature (Table 1) of a member of genus Actinoplanes producing an antibiotic of the kirromycin-like family.

Fig. 1. Actinoplanes sp. A8924: spherical and subspherical sporangia forming on the vegetative mycelium.

Stalks supporting the sporangia are visible. Light microscope $(\times 1,000)$.

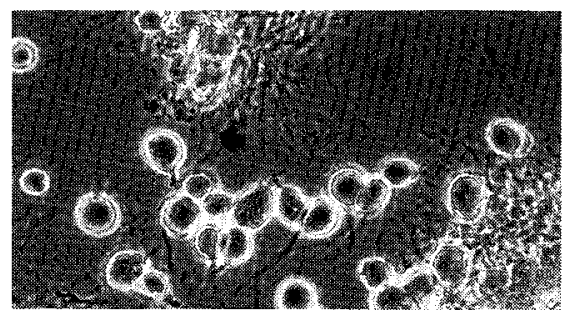

Table 1. Aurodox-type antibiotics.

\begin{tabular}{|c|c|}
\hline Antibiotic & Producer \\
\hline $\begin{array}{l}\text { Kirromycin } \\
\quad\left(\text { Mocimycin }^{20)} *\right)\end{array}$ & $\begin{array}{l}\text { Streptomyces ramocissumus } \\
\quad \text { CBS } 190-69 \\
\text { S. collinus Tu } 365\end{array}$ \\
\hline Aurodox ${ }^{5}$ & S. goldiniensis ATCC 21386 \\
\hline Heneicomycin ${ }^{21}$ ) & S. filipinensis NRRL 11044 \\
\hline SB22484 22$)$ & Streptomyces. sp. NRRL 15496 \\
\hline Efrotomycin ${ }^{23,24)}$ & $\begin{array}{l}\text { "Nocardia lactamdurans" } \\
\text { MA5887 } \\
\text { Nocardia } \text { sp. ATCC } 53758\end{array}$ \\
\hline Azdimycin ${ }^{25)}$ & $\begin{array}{l}\text { S. diastatochromogenes } \\
\text { ATCC } 31013\end{array}$ \\
\hline MSD $A 63 A^{26)}$ & S. hiroshimense ATCC 31586 \\
\hline Factumycin ${ }^{8)}$ & S. lavendulae ATCC 31312 \\
\hline$A 73 A^{7)}$ & S. viridifaciens ATCC 31495 \\
\hline UK6975327) & $\begin{array}{l}\text { Amycolatopsis orientalis } \\
\text { ATCC } 53550\end{array}$ \\
\hline $\mathrm{L}-681,217^{28)}$ & S. cattleya ATCC 39203 \\
\hline Phenelfamycin ${ }^{29)}$ & $\begin{array}{l}\text { S. violaceoniger } \\
\text { NRRL } 18084 \\
\text { S. violaceoniger } \\
\text { NRRL } 18920\end{array}$ \\
\hline LL-E $19020^{30)}$ & S. lydicus NRRL 18036 \\
\hline Dihydromocimycin ${ }^{31)}$ & $\begin{array}{l}\text { S. ramocissumus } \\
\text { CBS } 190-69\end{array}$ \\
\hline Kirrothricin ${ }^{9)}$ & S. cinnamoneus Tu 89 \\
\hline $\begin{array}{l}\text { New elfamycin } \\
\left(\mathrm{A} 83010 \mathrm{~F}^{33)}\right)\end{array}$ & $\begin{array}{l}\text { Streptomyces sp. CBS } 473.89 \\
\text { unidentified Actinomycetes }\end{array}$ \\
\hline
\end{tabular}

* Identical aurodox-type antibiotics described by different authors. 


\section{G. Beretta \\ F. LE MONNIER \\ E. SElva \\ F. MARinelli}

\section{MMDRI Lepetit Research Center, Gerenzano (Varese), Italy}

(Received February 8, 1993)

\section{References}

1) Frost, B. M.; M. E. Valiant, B. Weissberger \& E. L. Dulaney: Antibacterial activity of efrotomycin. J. Antibiotics 29: 1083 1091, 1976

2) Frost, B. M.; M. E. Valiant \& E. L. Dulaney: Antibacterial activity of heneicomycin. J. Antibiotics 32: $626 \sim 629,1979$

3) Swanson, R. N.; D. J. Hardy, N. L. Shipkowitz, C. W. Hanson, N. R. Ramer, L. J. Coen \& P. B. FERnANDES: Phenelfamycins, a novel complex of elfamycin-type antibiotics. III. Activity in vitro and in a hamster colitis model. J. Antibiotics 42: $94 \sim 101$, 1989

4) Berger, J.; H. H. Lehr, S. Teitel, H. Maehr \& E. GrunBERG: A new antibiotic X-5108 of Streptomyces origin. I. Production, isolation and properties. J. Antibiotics 26: 15 22, 1973

5) Hall, C. C.; J. D. Watkins \& N. H. GeorgopapadaKou: Effects of elfamycins on elongation factor $\mathrm{Tu}$ from Escherichia coli and Staphylococcus aureus. Antimicrob. Agents Chemother. 33: 322 325, 1989

6) Wolf, H. \& H. ZäHNER: Stoffwechselprodukte von mikroorganismen. 99. Mitteilung. Kirromycin. Arch. Mikrobiol. 83: 147 154, 1972

7) Dewey, R. S.; J. E. Flor, P. J. CASSidy, S. OMURA \& R. OrWA (Merck \& Co.): Discovery of A73A, a new efrotomycin-like antibiotic in fermentation broth. U.S. 4,262,002, Apr. 14, 1981

8) Gullo, V. P.; S. B. Zimmerman, R. S. Dewey, O. Hensens, P. J. Cassidy, R. Ōiwa \& S. Ōmura: Factumycin, a new antibiotic (A40A): Fermentation, isolation and antibacterial spectrum. J. Antibiotics 35: $1705 \sim 1707,1982$

9) Thein-SChranNER, I.; H. ZÄHNER, H.-U. HoPPE, I. HuMMEL \& A. ZeECK: Metabolic products of microorganisms. 209. Kirrothricin, a new member of the kirromycin group. J. Antibiotics 35: 948 956, 1982

10) BÉrdy, J.: Humidin-Type. In Handbook of Antibiotic Compounds. Vol. II. Ed., I. BÉRDY et al., pp. $347 \sim 354$, CRC Press Inc., 1980

11) Chacko, C. I. \& D. GotTlieb: The isolation of flavensomycin and humidin from the same strain of Streptomyces griseus and their antimicrobial properties. Phytopathology 55: $587 \sim 591,1965$

12) Otoguro, K.; A. Nakagawa \& S. Ōmura:
Setamycin, a 16-membered macrolide antibiotic identification and nematocidal activity. J. Antibiotics 41: $250 \sim 252,1988$

13) Ōmura, S.; K. Otoguro \& H. Tanaka: The mode of action of a novel 18-membered macrolide, virustomycin A (AM-2604 A), on Trichomonas foetus. J. Antibiotics 36: 1755 1761, 1983

14) Huang, L.; G. Albers-Schonberg, R. L. Monaghan, K. Jakubas, S. S. Pong, O. D. Hensens, R. W. Burg, D. A. Ostlind, J. Conroy \& E. O. STAPLEY: Discovery, production and purification of the $\mathrm{Na}^{+}, \mathrm{K}^{+}$, activated ATPase inhibitor, L-681,110 from the fermentation broth of Streptomyces $\mathrm{sp}$. MA-5038. J. Antibiotics 37: 970 975, 1984

15) Edwards, D. M. F.; E. Selva, S. Stella, L. F. Zerilli \& G. G. Galio: Mass spectrometric techniques for structure and novelty determination of kirromycin-like antibiotics. Biol. Mass Spectrometry 2l: $51 \sim 59,1992$

16) Maerz, A. \& M. R. Paul: A dictionaty of color. 2nd Edition, McGraw Hill Book Company Inc., New York, 1950

17) Staneck, J. L. \& G. D. Roberts: Simplified approach to identification of aerobic actinomycetes by thin-layer chromatography. Appl. Microbiol. 28: $226 \sim 231,1974$

18) Saddler, G. S.; P. Tavecchia, S. Lociuro, M. Zanol, L. Colombo \& E. SElva: Analysis of madurose and other actinomycete whole cell sugars by gas chromatography. J. Microbiol. Methods 14: $185 \sim 191,1991$

19) Lechevalier, M. P. \& H. A. Lechevalier: Chemical composition as a criterion in the classification of aerobic actinomycetes. Int. J. Syst. Bacteriol. 20: $435 \sim 443,1970$

20) Vos, C. \& J. Den Admiral (Koninklijke Nederlandsche): Antibiotic MYC 8003 and process for producing same. U.S. 3,927,211, Dec. 16, 1975

21) Zimmerman, S. B.; J. H. Chalmers, Jr., R. S. Dewey, E. O. Stapley \& S. Hernandez: Heneicomycin, a new antibiotic (A21A): Fermentation, isolation, and antibacterial spectrum. J. Antibiotics 32: 665 666, 1979

22) Selva, E.; G. Beretta, R. Pallanza, B. P. Goldstein, M. Berti, D. M. F. Edwards \& M. DENARO: Antibiotic SB22484: A novel complex of the aurodox group. I. Taxonomy of the producing organism, isolation of the antibiotics and chemical and biological characterization. J. Antibiotics 43: $1349 \sim 1358,1990$

23) Wax, R.; W. Maiese, R. Weston \& J. Birnbaum: Efrotomycin, a new antibiotic from Streptomyces lactamdurans. J. Antibiotics 29: 670 673, 1976

24) Jefson, M. R., J. Kanena, S. Nishiyama \& J. TONE (Pfizer): Production of $N$-demethyl-efrotomycin by microbial glycosylation of mocimycin, using new efrotomycin-producing Nocardia species. U.S. 4,937,184-A, June 26, 1990 
25) Nimeck, M. W.; E. Meyers \& W. LiU (Squibb \& Sons Inc.): Antibiotic azdimycin. U.S. 3,898,327, Aug. 5, 1975

26) Hernandez, S.; S. B. Zimmerman, V. P. Gullo \& R. S. DEWEY (Merck \& Co.): Discovery of MSD A63A, a new efrotomycin-like antibiotic in fermentation broth. U.S. 4,311,693, Jan. 19, 1982

27) Pacey, M. S.; M. R. Jefson, L. H. Huang, W. P. Cullen, H. Maeda, J. Tone, S. Nishiyama, K. KANEDA \& M. IsHIGURO: UK-69,753, a novel member of the efrotomycin family of antibiotics. I. Taxonomy of the producing organism, fermentation and isolation. J. Antibiotics 42: 1453 1459, 1989

28) Kempf, A. J.; K. E. Wilson, O. D. Hensens, R. L. Monaghan, S. B. Zimmerman \& E. L. Dulaney: L-681,217, a new novel member of the efrotomycin family of antibiotics. J. Antibiotics 39: $1361 \sim 1367$, 1986

29) Hochlowski, J. E.; M. H. BuytendorP, D. N. Whittern, A. M. Buko, R. H. Chen \& J. B. MCALPINE: Phenelfamycins, a novel complex of elfamycin-type antibiotics. II. Isolation and structure determination. J. Antibiotics 41: 1300 1315, 1988

30) Carter, G. T.; D. W. Phillipson, J. J. Goodman, T. S. DunNe \& D. B. Borders: LL-E19020 $\alpha$ and $\beta$, novel growth promoting agents: Isolation, characterization and structures. J. Antibiotics 41: $1511 \sim 1514$, 1988

31) Jongsma, H. M; H. J. Kooreman \& J. L. Os (Gist-Brocades, N.V., Delft (Netherland)): Dihydromocimycin, dessen Sälze mit Säuren, Verfahren zu seiner Herstellung und Futtermittel. Offenlegungsschrift 2,621,615, Nov. 25, 1976

32) MargrafF, R; T. Kiener \& A. Kies (RhonePoulenc): Nouvelle elfamycine, son procédé de préparation et son utilisation. Eur. Pat. Appl. 442,783 Al., Feb. 7, 1991

33) Smitka, T. A.; R. Bonjouklian, T. J. Perun, Jr., A. H. Hunt, L. D. BoECK \& R. C. YAO: A83016F, a new member of the aurodox family. J. Antibiotics 45: $433 \sim 443,1992$ 